\title{
Source of the midline echo and its implications in echoencephalography
}

\author{
T. R. FISHER \\ From the Department of Neurological Surgery and the \\ Accident Service, Radcliffe Infirmary, Oxford
}

Ultrasound as a technique in neurological diagnosis was introduced by Leksell in 1955, and is now gaining acceptance as a useful screening test for intracranial tumours and haematomas. The technique uses a pulsed ultrasonic beam transmitted from a probe (transducer) applied to the temporal region of the skull. The ultrasound is then reflected from a 'midline structure' within the cranium. Spaceoccupying lesions are suspected when the midline echo is displaced laterally.

Although the method is simple to use, errors in interpreting the reflected midline echo occur. Clinical studies by Lithander (1960), Ford and Ambrose (1963), and Brinker, King, and Taveras (1965) record false midline displacements in 6 to $12 \%$ of cases and normal midline echos associated with spaceoccupying lesions in 0.5 to $5.7 \%$. Failure in technique is partly responsible, as these authors have shown. However, in some situations where bilateral chronic subdural haematomas or bitemporal lobe contusions have balanced each other, or, as Lithander (1961) has shown, when moderate unilateral extracerebral haematomas have collected high over the convexity of the hemisphere, there may be no displacement of the midline echo, whereas a unilateral frontal mass, although displacing the anterior structures locally, need have no effect on those more posteriorly situated. In view of these interpretive difficulties the generalized term 'midline echo' appeared unsatisfactory. Indeed as the midline echo could be constantly produced with similar characteristics in different individuals it was probable that the echo signal did occur from a specific anatomical structure. Its identity has been previously investigated and variously held to be the longitudinal fissure (de Vlieger and Ridder, 1959), septum lucidum (Gordon, 1959), third ventricle (Ford and Ambrose, 1963), pineal body (Leksell, 1958; Jeppsson, 1961; Brylski and Izenstark, 1965), and structures in the region of the posterior part of the third ventricle (Brinker et al., 1965).

Evidence from the present study into the reflecting source of the midline echo favours the pineal body.

\section{ECHOENCEPHALOGRAPHY}

Ultrasonic echoencephalography uses a pulsed highfrequency wave form of usually $1.5 \mathrm{mc}$./ $/ \mathrm{sec}$., transmitted from a transducer consisting of barium titanate crystals, that acts both as a transmitter and receiver. It requires a medium, either liquid or solid, for its propagation, and because of its high frequency the ultrasonic beam has properties, similar to light, of reflection, refraction, absorption, and dispersion. If the ultrasonic beam passes from one medium to another of differing acoustic impedance (defined as the product of the density and the velocity of sound through that medium) part of it is reflected from the interface between the two mediums. The echo thus formed is picked up by the transducer and relayed onto an oscillograph screen. A greater proportion of the ultrasound is reflected if the acoustic impedance of the medium is high. Likewise the amplitude of the echo signal is increased if the interface is large rather than small, and if the ultrasonic beam is transmitted directly onto the interface rather than at an angle.

\section{CLINICAL TECHNIQUE}

POSITION OF TRANSDUCER AND RECOGNITION OF THE MIDLINE ECHO The reflected midline echo was picked up by a transducer applied to the temporal region of the skull. There were three verticals, in front of, above, and behind the ear, on which the transducer was able to receive reflected midline echos. On the ear vertical the reflected echo was constantly and the most easily received. But recordings taken from in front of, or behind, the ear were frequently unsatisfactory. However, in these positions if the transducer were tilted such that the transmitted ultrasonic beam intersected the line joining the two opposite ear vertical positions, then often a good reflected echo was received.

The echo signal observed in the midline was the strongest and most persistent of the echos within the cranium and remained the most constant while rocking the transducer. It appeared on the oscillograph as a single or notched spike, or double spike varying with the tilt of the probe. Occasionally it was the central echo in a complex of three, and characteristically the amplitude of the echo signal might pulsate. 


\section{INVESTIGATION}

Attention was directed to localizing the anatomical structures within the ultrasonic beam after it had picked up the dominant midline echo and relating these to the position of the transducer on the skull surface. Clinical observations were correlated with experiments using post-mortem material. The reflecting brain-cerebrospinal fluid interface was investigated using dissected pineal bodies.

EXPERIMENT 1 A post-mortem skull was sectioned in the horizontal plane just above the ears and the brain removed. This allowed examination of the two lateral walls of the cranial cavity and their relationship to each other.

It was found that at a point level with the ears, the two temporal bones were most nearly parallel. From this point the skull curved forwards and medially at an angle varying between $10^{\circ}$ and $40^{\circ}$ and curved backwards and medially at an angle between $20^{\circ}$ and $50^{\circ}$.

EXPERIMENT 2 As the midline echo was most constantly and easily produced from the ear vertical position, structures related to this beam were investigated.

A cadaver was set so that the cranium lay vertically above the trunk. The calvarium was removed, as for a routine necropsy, exposing the cerebral cortex. A line taken from a point above each pinna was marked onto the cerebral hemispheres. After removal of the brain, it was fixed in $10 \%$ formaldehyde for one week. The brain was then sectioned along this line (in the coronal plane). This section was found to pass behind the third ventricle showing in the midline the base of the pineal body, the fibres of the posterior commissure, and the acqueduct of Sylvius before it passes beneath the superior colliculi.

EXPERIMENT 3: LOCALIZATION OF THE DOMINANT ECHO (IN A FIXED BRAIN) A brain was removed from a cadaver and fixed in $10 \%$ formaldehyde for three weeks. It was then transferred to a plastic chamber filled with $20 \%$ dextrose. The brain, while submerged in this chamber, was radiographed to exclude any intracranial air. The transducer was positioned alternately along the walls of the chamber, transmitting a beam in the lateral and antero-posterior directions. This picked up a strong echo from a source that coincided with the intersection of the beam from each direction. The depth at which the echo appeared was recorded. The fixed brain was sectioned in a coronal plane at the measured distance, with a probe previously inserted in the midline to the requisite depth.

It was found that this coronal section passed through the pineal body which lay at the point of the probe.

EXPERIMENT 4: PINEAL BODY AS A SOURCE OF THE MIDLINE ECHO The midline structures in relation to the third ventricle were removed in a block from a fixed brain. The roof of the third ventricle was removed exposing the third ventricle, the pineal body posteriorly, and the lateral walls of the quadrigeminal cistern.

By submerging this bloc in a chamber, echos could be obtained from a transverse direction from both the walls

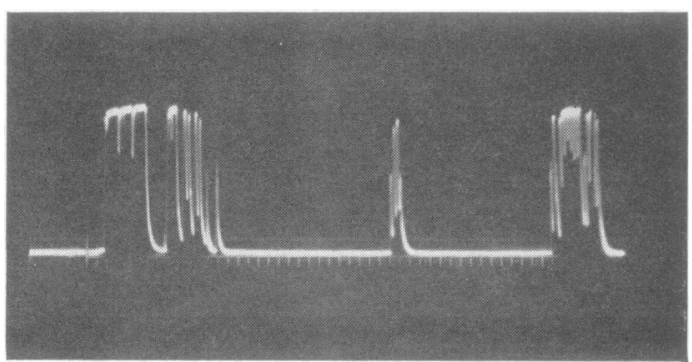

FIG. 1. The echo produced from the third ventricle from a transverse beam.

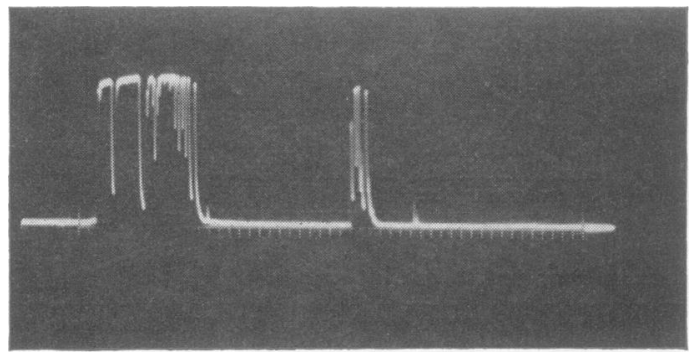

FIG. 2. The echo produced from the pineal body with transverse beam.

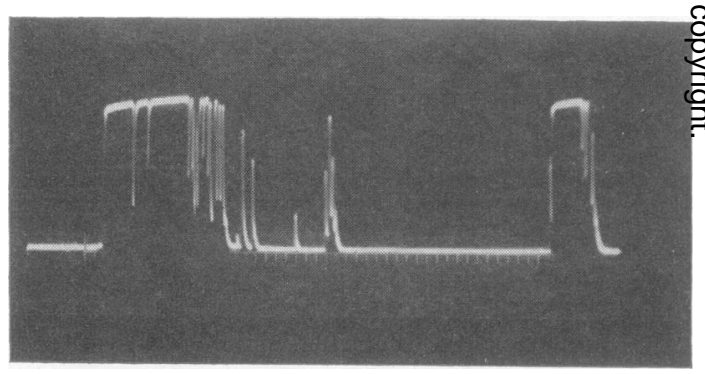

FIG. 3. The echo produced from the pineal body from a postero-anterior beam.

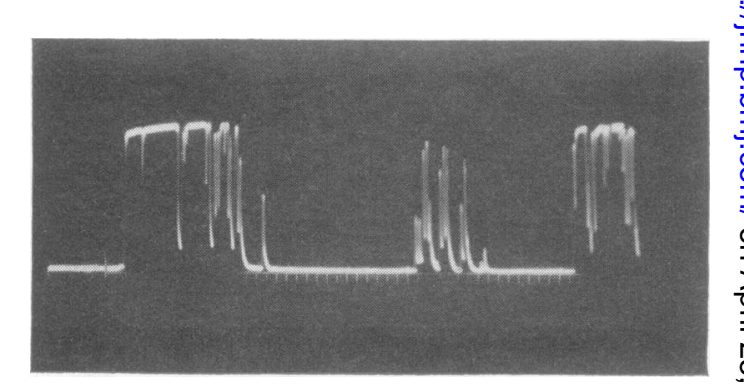

FIG. 4. The complex three spikes produced by the pineal

body and the lateral walls of the quadrigeminal cistern. 
of the third ventricle (Fig. 1) and the pineal body (Fig. 2). It was noted that occasionally a complex of three spikes was obtained (Fig. 4), the central spike being reflected from the pineal body and the lateral spikes from the lateral walls of the quadrigeminal cistern. A single echo of good amplitude was also produced in the posteroanterior direction (Fig. 3).

To confirm the localization of the echo to each individual structure, a metal probe was passed over that structure which either increased the existing echo or displaced it. In addition, by passing a thin thread through the pineal body (the thread itself too small to produce a significant echo) and by pulling the thread, the pineal echo was seen to fluctuate with each change in position.

EXPERIMENT 5: INTERFACE NECESSARY FOR PRODUCTION OF THE MIDLINE ECHO The evidence collected in the previous experiments suggested that the pineal body was the source of the midline echo. The echo could have been reflected from the interface either between brain tissue and cerebrospinal fluid or between brain tissue and calcium deposits in the pineal body.

1 Eighteen pineal bodies were dissected from cadavers between the ages of 20 and 80 . Half were fixed in formaldehyde and half were left in a fresh state. Both groups were radiographed and in those pineal bodies studied all contained radiological or histological evidence of calcium deposits. The sections were stained for calcium by the Von Kossa technique.

2 Both groups were placed in a chamber of varying concentrations of dextrose, such that each lay suspended in the fluid beneath the surface. The transducer picked up a good echo from each.

3 Both groups were now decalcified, and the same procedure was carried out (all gave a good echo). A gland from each group was sectioned and stained for calcium to confirm satisfactory decalcification, after suspension in $10 \%$ formic acid for three days.

4 Decalcified pineal bodies were floated in a similar manner and it was found that the amplitude of the echo signal increased with the size of the piece of tissue, i.e., the area of the presenting interface.

5 By changing by a few degrees the angle of incidence of the beam of ultrasound on an individual pineal body a single spike, notched, or double spike could be produced.

EXPERIMENT 6: ANATOMICAL CEREBROSPINAL FLUID SPACE AROUND THE PINEAL BODY The pineal body lay in the quadrigeminal cistern, but was overlapped anteriorly by the cerebrospinal fluid in the suprapineal recess. By removing the corpus callosum of a fixed brain the lateral boundaries of the quadrigeminal cistern were seen to be equidistant from the pineal body at its tip and measured $0.75 \mathrm{~cm}$. on each side. As the lateral boundaries run obliquely they were closer to the pineal body anteriorly and further apart posteriorly.

\section{CONCLUSIONS}

On clinical examination the midline echo was best reflected from the ear vertical position. A coronal section of the brain from the ear vertical position passed through the pineal body in the midline. The dominant echo is a fixed brain was found to coincide with the pineal body. Under direct vision the pineal body reflected a good echo signal. The echo reflected occurred from the pineal tissue-cerebrospinal fluid interface rather than the pineal-calcium interface. The echo reflected from the pineal body had similar characteristics to the midline echo observed on clinical examination. The pineal body is surrounded by a large volume of cerebrospinal fluid.

\section{DISCUSSION}

The problem of the source of the midline echo resolves around the question of why the dominant echo should be only picked up in the temporal region. This could be explained either because of special structural or environmental characteristics of the median structure or from physical limitations set up by the skull for positioning the probe, such as thickness and curvature. The first possibility is unlikely as in practice and experimentally any structure with a brain-cerebrospinal fluid interface can reflect an echo. For this reason echos have been reflected under experimental conditions from the longitudinal fissure, the septum lucidum, the third ventricle, and the pineal body. The second possibility, that of skull curvature, determines, I think, which of these structures reflects the characteristic echo in echoencephalography. Except for the pineal body, the other three midline structures - the longitudinal fissure, the septum lucidum, and the third ventricle - are all flat vertical surfaces so that for a transducer to pick up a reflected echo the bone surface of the skull would have to be parallel to the structure in the midline. In experiment it was found that the only skull surface parallel to the midline was in the temporal region related to the superior aspect of the pinna (the ear vertical position). Experiment 2, by showing that a coronal section from this position passes behind the third ventricle, made it unlikely that either the third ventricle or the septum lucidum would normally reflect an echo. The third ventricle, however, is in close proximity anteriorly to the pineal body. Even so it is unlikely to reflect an echo, for a transducer positioned, other than in the ear vertical position, will be tilted by the natural curvature of the skull causing the ultrasound beam to be transmitted at an angle to the median plane.

Clinical observation that the optimum position for the transducer was close to the superior aspect of the ear, and that the structure lay within the ultrasonic beam transmitted from this position, is confirmed from a study by Brylski and Izenstark (1965). They attached a transducer to an arc- 
template and took positional readings on either side of the skull after beaming ultrasound at differing incidences to the midline structure. By joining opposite skull markers across a lateral view of a pneumoencephalogram they were seen to intersect behind the third ventricle through the region of the pineal body. These lines repeated on a plain view of the skull with a calcified pineal body were found to intersect through this structure. Brinker et al. (1965), after examining angiographic records and correlating the shift of the midline vessels with the degree of shift found with the ultrasound technique, came to the conclusion that the more posterior the structure, the better the correlation between the two techniques. The pineal body was credited with the closest correlation.

One of the characteristics of the structure producing the midline echo was suggested when, by tilting the transducer in front or behind the ear, the midline echo could still be received. It introduced the conception that this structure was either round or oval and not a single vertical interface, as this would deflect rather than reflect echos if the incident beam was more than a few degrees from normal. This has additional support from the known practice that by rocking the transducer the amplitude of all other echos, except the dominant midline echo, are decreased.

With strong presumptive evidence from these early tests that the pineal body was the source of the midline echo Jeppsson's (1961) exploration of a brain submerged in a chamber was repeated. His findings that the dominant echo coincided with the pineal body were confirmed. By extending the study to direct observation, the pineal body was seen to reflect an echo of large amplitude in both lateral and antero-posterior directions. Contrary to Jeppsson's (1961) findings, the interface reflecting the pineal echo was between brain and cerebrospinal fluid rather than brain and calcium deposits. That there was a large cerebrospinal fluid space around the pineal body was apparent on examination of the boundaries of the quadrigeminal cistern. This is confirmed by the work of Di Chiro (1961) with air studies of the third ventricle and Liliequist (1959) with similar studies of the quadrigeminal cistern.

A transducer placed in front or behind the ear will reflect an echo only from the pineal body because of its oval shape and the normal skull curvature. But in a few cases when the temporal bones present a larger surface parallel to each other, then the third ventricle and the septum lucidum in front could reflect midline echo. This situation rarely occurs in adults except in dolichocephalic skulls but commonly in children where the lateral walls of the cranial cavity are much more nearly parallel than in $\underset{\mathbb{D}}{Z}$ the adult. Possibly because of a similar situation Lithander (1961) was able to record two midline echos from a patient. He found a normal midline $\widehat{G}$ echo from a position above the ear, but marked 0 lateral displacement with the transducer placed in front of the ear. This was shown during angiography to be caused by a frontal mass, which had produced distortion of the anterior vessels but not of the posterior vessels. This introduced the possibility of $\overrightarrow{\vec{F}}$ placing a 'stand off' attachment to the transducer. $\overrightarrow{0}$ It would eliminate any skull curvature and allow $\frac{}{0}$ routine examination to include scanning of the pineal $\frac{\bar{\sigma}}{\bar{D}}$ body and the posterior and anterior parts of the $\overrightarrow{\mathbb{D}}$ third ventricle, so that previously concealed lesions $\frac{2}{0}$ of the fronto-temporal areas could be identified.

\section{SUMMARY}

A good echo can be reflected from any brain tissuecerebrospinal fluid interface. But the source of the midline echo in echoencephalography is the surface iv of the pineal body. It is limited to this structure by of the normal curvature of the skull, which tilts the transducer unless placed in the ear vertical position $v$ At this point the two temporal bones are mos nearly parallel. With larger parallel skull surfaces more than one midline echo could possibly be obtained. It is suggested that a 'stand off' attach $\frac{O}{0}$ ment to the transducer might extend echoencephale graphic diagnosis.

I am indebted to Mr. J. M. Potter for his help and sugges- क tions in preparing this paper.

\section{REFERENCES}

Brinker, R. A., King, D. L., and Taveras, J. M. (1965). Echoencephalography. Amer. J. Roentgenol., 93, 781-790.

Brylski, J. R., and Izenstark, J. L. (1965). Anatomic localization of midline echo in sonograms of the brain. Ibid., 93, 811-815.

de Vlieger, M., and Ridder, H. J. (1959). Use of echoencephalography. Neurology (Minneap.), 9, 216-223.

Di Chiro, G. (1961). An Atlas of Detailed Normal Pneumoencephalo graphic Anatomy, pp. 86-90. Thomas, Springfield, Illinois.

Ford, R., and Ambrose, J. (1963). Echoencephalography. Brain, 86, 189-196.

Gordon, D. (1959). Echo-encephalography: ultrasonic rays in diagnostic radiology. Brit. med. J., 1, 1500-1504.

Jeppsson, S. (1961). Echoencephalography IV. The midline echo: an evaluation of its usefulness for diagnosing intracranial expansivities and an investigation into its sources. Acta chir. scand., suppl., 272, 1-151.

Leksell, L. (1955). Kirurgisk Behandling av akuta skallskador. Nord. Med., 54, 1444-1446.

- (1958). Echoencephalography II. Midline echo from the pineal body as an index of pineal displacement. Acta chir. scand., 115, 255-259.

Lithander, B. (1960). The clinical use of echo-encephalography. Acto psychiat. scand., 35, 241-244.

- (1961). Origin of echoes in the echoencephalogram. J. Neurol. U Neurosurg. Psychiat., 24, 22-31.

Liliequist, B. (1959). Subarachnoid cisterns-an anatomic and $N$ roentgenologic study. Acta radiol (Stockh.), suppl., 185, 80-86. 